\title{
Electroacupuncture Alleviates Depressive-Like Symptoms and Modulates BDNF Signaling in 6-Hydroxydopamine Rats
}

\author{
Min Sun, ${ }^{1}$ Ke Wang, ${ }^{1}$ Yan Yu, ${ }^{1}$ Wen-Ting Su, ${ }^{1}$ Xin-Xin Jiang, \\ Jian Yang, ${ }^{2}$ Jun Jia, ${ }^{1}$ and Xiao-Min Wang ${ }^{1}$ \\ ${ }^{1}$ Departments of Neurobiology and Physiology, Key Laboratory for Neurodegenerative Disorders of the Ministry of Education, Beijing \\ Key Laboratory for Parkinson's Disease, Beijing Institute for Brain Disorders, Capital Medical University, Beijing 100069, China \\ ${ }^{2}$ Beijing Key Laboratory for Mental Disorders, China Clinical Research Center for Mental Disorders, Beijing Anding Hospital, \\ Capital Medical University, 5 Ankang Alley, Beijing 100088, China
}

Correspondence should be addressed to Jian Yang; kevinyangj@sina.com, Jun Jia; jiajun@ccmu.edu.cn, and Xiao-Min Wang; xmwang@ccmu.edu.cn

Received 10 April 2016; Accepted 16 June 2016

Academic Editor: Pierre Champy

Copyright (c) 2016 Min Sun et al. This is an open access article distributed under the Creative Commons Attribution License, which permits unrestricted use, distribution, and reproduction in any medium, provided the original work is properly cited.

\begin{abstract}
Previous studies have identified the beneficial effects of electroacupuncture (EA) on motor behaviors in Parkinson's disease (PD). However, the role and potential mechanisms of EA in PD-associated depression remain unclear. In the present study, a rat model of PD with unilateral 6-hydroxydopamine (6-OHDA) lesions in the medial forebrain bundle was treated using EA for 4 weeks. We found that $100 \mathrm{~Hz}$ EA improved several motor phenotypes. In addition, tyrosine hydroxylase (TH) immunohistochemical analysis showed that EA had a minimal impact on the TH-positive profiles of the ipsilateral ventral tegmental area. Compared with the 6-OHDA group, long-term EA stimulation significantly increased sucrose solution consumption and decreased immobility time in the forced swim test. EA treatment did not alter dopamine, norepinephrine, and serotonin levels in the striatum and hippocampus. Noticeably, EA treatment reversed the 6-OHDA-induced abnormal expression of brain-derived neurotrophic factor (BDNF) and tropomyosin-related kinase $\mathrm{B}(\mathrm{TrkB})$ in the midbrain and hippocampus. These results demonstrate that EA at $100-\mathrm{Hz}$ possesses the ability to improve depressive-like symptoms in PD rats, which is, at least in part, due to the distinct effect of EA on the mesostriatal and mesocorticolimbic dopaminergic pathways. Moreover, BDNF seems to participate in the effect of EA in PD.
\end{abstract}

\section{Introduction}

Parkinson's disease (PD) is generally recognized as a progressive neurodegenerative disorder characterized by motor dysfunction. However, accumulating evidence indicates that nonmotor symptoms of PD, such as sensory dysfunction, sleep disturbance, and psychiatric complications (depression, anxiety, apathy, and cognitive impairment), are more detrimental to well-being and functional ability than motor symptoms [1-3]. As one of the most common comorbidities, depression affects approximately $40-50 \%$ of PD patients [4] and might account for substantial disability and poor quality of life. Currently, selective serotonin reuptake inhibitors and tricyclic antidepressants are the two main categories of antidepressants used for treating depression in PD. However, the efficacy of antidepressant treatment in PD remains unclear [5]. Besides, polypharmacy, with antidepressant and antiparkinsonian treatments, may produce unexpected drug interactions and complicate disease development [6].

Acupuncture, a traditional complementary and alternative medical approach, has been widely used as an adjunct to standard therapy for PD. Although the definite role of acupuncture in PD is controversial [7], increasing evidence shows that acupuncture or electroacupuncture (EA) can alleviate motor and nonmotor symptoms, including tremors, slowness, pain, depression, sleep disturbance [8], and anxiety [9], and is particularly effective at early stages in PD patients [10]. Prior experiments have indicated that high-frequency EA stimulation $(100 \mathrm{~Hz})$ is effective in improving motor function in various animal models of PD by normalizing neurotransmitters in the basal ganglia [11], inhibiting neuroinflammatory responses [12], reducing oxidative stress [13, 
14], and increasing neurotrophic factors [15], among other methods of action. However, few studies have investigated the exact effects of EA on nonmotor symptoms, particularly depressive symptoms.

Brain-derived neurotrophic factor (BDNF), a member of the neurotrophin family, plays critical roles in cell differentiation, neuronal survival, migration, and synaptic plasticity. $\mathrm{BDNF}$ is reportedly involved in the pathophysiology of both $\mathrm{PD}$ and depression, as evidenced by the fact that altered expression of BDNF mRNA and protein has been observed in postmortem studies of PD patients [16]. Meanwhile, reduced expression of BDNF mRNA and protein has been found in the mesostriatal and mesocorticolimbic pathways, as well as in the serum of patients with depression $[17,18]$. Further alterations in BDNF expression have been observed in the mesocorticolimbic and nigrostriatal systems in experimental animals [19-22]. BDNF needs to bind to its high-affinity protein kinase receptor, tropomyosin-related kinase $\mathrm{B}(\operatorname{Trk} \mathrm{B})$, to exert its biological effects [23]. Although the precise mechanism is under investigation, abnormalities in BDNF-TrkB signaling may substantially contribute to the development of depression in PD.

Animal models are an important way to improve our understanding of the pathophysiological mechanisms of PD and possible treatments. Studies have suggested that bilateral infusion of 6-hydroxydopamine (6-OHDA) into the striatum [24], SNc [25, 26], or ventral tegmental area (VTA) [27, 28] produces a depressive-like phenotype in rats. Among PD models, the rat model of a unilateral 6-OHDA lesion in the medial forebrain bundle (MFB) has lesions in both the mesostriatal and mesocorticolimbic dopaminergic pathways $[29,30]$. This lesion protocol is known to cause significant motor features and several nonmotor impairments of PD, including depression, apathy, and anhedonia [31]. In the present study, we evaluated the effect of EA treatment on both motor and nonmotor behaviors in the unilateral MFBlesioned PD rat model. We have also investigated the changes of these neurotransmitters including dopamine (DA), norepinephrine (NE), and serotonin (5-HT) in the striatum and hippocampus after 4 weeks of EA treatment. In addition, the effects of EA treatment on BDNF-TrkB signals within the midbrain and hippocampus were examined. The purpose of this study is to explore the effects of EA treatment on the motor and nonmotor phenotypes and elucidate the potential antidepressive mechanism mediated by EA in PD.

\section{Methods}

2.1. Animals and Unilateral 6-OHDA Lesion Induction. Adult Sprague-Dawley (SD) rats weighing 200-220 g were obtained from the animal facility of Capital Medical University. Rats were housed three per cage under standard laboratory conditions: 12 -h light/dark cycle, $22^{\circ} \mathrm{C}$ room temperature, $55 \%$ relative humidity, and access to food and water ad libitum. All experimental procedures were performed according to the Ethics Committee on Animal Care and Usage of Capital Medical University.
Rats were anesthetized with $2 \%$ pentobarbital sodium ( $40 \mathrm{mg} / \mathrm{kg}$, i.p.) and fixed into a Kopf stereotaxic apparatus. Lesions were induced by injection of 6-OHDA using a 30-gauge needle Hamilton syringe into the right $\mathrm{MFB}$ at the following coordinates: anteroposterior (AP), $-4.3 \mathrm{~mm}$; mediolateral (ML), $-1.5 \mathrm{~mm}$; and dorsoventral (DV), $7.6 \mathrm{~mm}$ from the dura. A total dose of $8 \mu \mathrm{g}(5 \mu \mathrm{g} / \mu \mathrm{L}, 1.6 \mu \mathrm{L}) 6-\mathrm{OHDA}$ (Sigma-Aldrich) was administered, at a rate of $0.5 \mu \mathrm{L} / \mathrm{min}$. The microsyringe was left in place for $4 \mathrm{~min}$ to allow diffusion. Sham-operated rats underwent the same surgical procedure but received saline injection. Behaviors were assessed 2 weeks after surgery.

2.2. EA Stimulation. EA stimulation was administered from 2 weeks to 6 weeks after 6-OHDA lesions were induced in the rats. Rats were randomly divided into 4 groups: a vehicletreated sham group, a 6-OHDA-lesioned model group, and two groups of 6-OHDA-lesioned rats receiving EA at either 0 or $100 \mathrm{~Hz}$. Two stainless steel needles of diameter $0.25 \mathrm{~mm}$ were inserted at a depth of $5 \mathrm{~mm}$ into the acupoints of BAIHUI (GV 20, at the midpoint between the auricular apices) and DAZHUI (GV 14, directly below the spinous process of the vertebra prominens). A bidirectional square wave $(0.2 \mathrm{~ms})$ electrical pulse with frequency $100 \mathrm{~Hz}$ was administered from a medical EA apparatus (HANS, Neuroscience Research Institute, Beijing, China) for $30 \mathrm{~min}$ a day, 6 days a week for 4 weeks. The intensity of stimulation was increased stepwise from 1 to $2 \mathrm{~mA}$ and then to $3 \mathrm{~mA}$, with each step lasting for $10 \mathrm{~min}$. During EA stimulation, the rats were kept in the cage in an awake, unrestrained condition. Those treated with EA at $0 \mathrm{~Hz}$ underwent the same procedures, but no electrical pulses were delivered through the needles.

\subsection{Behavioral Assessment}

2.3.1. Rotarod Test. The rotarod test was used to evaluate balance and motor coordination. The initial 3 days of testing served as training, and rats were placed on the rod turning at a low speed, permitting them to attain stable performance. On the test day, an accelerating protocol going from $4 \mathrm{rpm}$ to $40 \mathrm{rpm}$ in $2 \mathrm{~min}$ was performed, and the time taken for the rats to fall was automatically recorded.

2.3.2. Open Field Test. To assess general locomotor activity and anxiety-related activity, the rats were monitored using automatic infrared beams in a black chamber (TruScan 2.0 Instruments, Columbus, $\mathrm{OH}$ ). The rats were placed in the arena and allowed to freely move about for $30 \mathrm{~min}$ while being recorded by an overhead camera. The footage was then analyzed using DigiScan analyzer and software (TruScan 2.0, Columbus) to extract the following parameters: horizontal movement distance, vertical movement distance, and numbers of entries into the arena center.

2.3.3. Apomorphine-Induced Turning Behavior. This behavioral test was performed in a blinded fashion. The rats 
received a subcutaneous injection of $0.05 \mathrm{mg} / \mathrm{kg}$ apomorphine hydrochloride (Sigma) dissolved in $1 \%$ ascorbic acid and $0.9 \% \mathrm{NaCl}$. Immediately after apomorphine injection, the net number of rotations was recorded and assessed over $30 \mathrm{~min}$. Rotational testing was performed in automatic rotameter bowls (Coulbourn Instruments Inc., PA, USA).

2.3.4. Sucrose Preference Test. The sucrose preference test is used as an indicator of anhedonic behavior in rodents. Before the beginning of testing, rats were kept individually habituated to two needleless syringes (filled with $1 \%$ sucrose solution and plain water, resp.) $8 \mathrm{~h}$ per day for 2 days in their home cage. After training, the rats were deprived of food and water for $12 \mathrm{~h}$ and then exposed to the two syringes for 30 min as described previously [32]. After an interval of $1 \mathrm{~h}$, the positions of the syringes were exchanged, and the rats were tested for $30 \mathrm{~min}$ again. The volume of water and sucrose solution intake during $1 \mathrm{~h}$ was measured. Sucrose preference was calculated as a percentage of the volume of sucrose intake to the total volume of fluid intake.

2.3.5. Forced Swim Test. The forced swim test (FST) is commonly used to assess behavioral despair in rodents [22, 23]. The protocol consists of a 15-min pretest swim and a 5-min test swim on the following day. Rats were placed into a cylindrical receptacle (height, $60 \mathrm{~cm}$; diameter, $26 \mathrm{~cm}$ ) filled with water $\left(25^{\circ} \mathrm{C}, 35 \mathrm{~cm}\right.$ deep $)$. Behavioral activity was recorded for $5 \mathrm{~min}$ using a digital video camera connected to SMART video-tracking system (Panlab, Spain). The amount of time spent in a posture of immobility (i.e., the lack of motion of the entire body, with the exception of only small movements necessary to keep the animal's head above the water), swimming (i.e., large forepaw movements that displaced water and moved the animal's body around the cylinder, which were more than necessary to keep the head above the water), and climbing (i.e., vigorous movements of the forepaws in and out of the water, usually directed against the wall of the tank) was calculated. All the behavioral tests were performed following randomization and blinding.

\subsection{TH Immunohistochemical Analysis and Quantification.} Rats were sacrificed and transcardially perfused with $4 \%$ paraformaldehyde in $0.1 \mathrm{M}$ phosphate buffer. Frozen mesencephalon coronal sections were cut into $30-\mu \mathrm{m}$ thick slices on a cryostat and processed for $\mathrm{TH}$ immunohistochemical analysis. Brain slices were incubated overnight at $4^{\circ} \mathrm{C}$ with TH antibody (diluted 1:2000, Chemicon, USA), followed by incubation with a biotinylated secondary antibody (Lab Vision, USA) for $30 \mathrm{~min}$. The antibody was detected with an avidine/biotine complex (Vector Laboratories, USA) and visualized with 3,3-diaminobenzidine (Sigma). Six identical TH-labeled slices spanning the entire mesencephalon and including both SNc and VTA brain regions were selected from 5 animals per group. Using a bright-field microscope (Olympic, Japan), the boundaries of the SNc and VTA areas were drawn. The number of $\mathrm{TH}$-immunoreactive profiles in these areas was counted by stereology, using Stereo Investigator software (MBF Bioscience, USA) as described previously [33]. The survival percentage of TH-positive cells was calculated as a percentage of the number of surviving cells on the lesioned side to that on the nonlesioned side. An independent investigator evaluated all sections in a blinded manner.

2.5. Quantification of DA, 5-TH, and NE. Rats were sacrificed by decapitation 1 day after behavioral measurements. The striatum, ventral midbrain, and hippocampus were rapidly dissected and stored in liquid nitrogen at $-80^{\circ} \mathrm{C}$. The concentrations of DA, 5-HT, and NE were assayed using reverse-phase high-performance liquid chromatography (HPLC) with electrochemical detection. The tissue samples were homogenized with an ultrasonic cell disrupter (Sonics) in $0.4 \mathrm{M}$ perchloric acid. After centrifugation at $10,000 \times \mathrm{g}$ for $30 \mathrm{~min}$, the supernatant $(160 \mu \mathrm{L})$ was mixed with $80 \mu \mathrm{L}$ mobile phase solution and was kept in the dark in an ice-bath $(60 \mathrm{~min})$, followed by centrifugation (12,000 rpm, $20 \mathrm{~min})$. The resultant supernatant was injected into the chromatograph. The mobile phase solution (flow rate, $1 \mathrm{~mL} / \mathrm{min}$ ) comprised $63.5 \mathrm{mM}$ citric acid monohydrate, $60.9 \mathrm{mM}$ trisodium citrate dihydrate, $0.1 \mathrm{mM}$ EDTA, $0.5 \mathrm{mM}$ 1-octanesulfonic acid sodium salt, and $8 \%$ methanol. The $\mathrm{pH}$ of the running buffer solution was adjusted to 4.0 , and it was then filtered through a filter of pore size $0.45 \mathrm{~mm}$. The peak areas of external standards were used to quantify sample peaks.

2.6. Enzyme-Linked Immunosorbent Assay. The level of BDNF was determined by enzyme-linked immunosorbent assay (ELISA). The ventral midbrain and hippocampus were rapidly dissected and homogenized in a lysis buffer containing protease inhibitors (Sigma-Aldrich). The sample protein contents were measured using a BCA protein assay kit (Pierce, Rockford, IL). For ELISA, a BDNF ELISA kit was used according to the manufacturer's instructions (Millipore, Billerica, MA). BDNF levels were determined relative to a standard curve constructed using BDNF protein standards that were assayed simultaneously with the experimental samples.

2.7. Western Blotting. The expression of TrkB was determined by western blotting. The ventral midbrain and hippocampus tissues of rats were prepared as described previously [32]. Equivalent amounts of proteins were processed for SDSPAGE and electrotransferred onto Immobilon-P membranes (Millipore, Billerica, MA). After they were blocked with 5\% nonfat milk in $0.5 \%$ Tween 20 in $20 \mathrm{mM}$ Tris and $137 \mathrm{mM}$ $\mathrm{NaCl}$ for $1 \mathrm{~h}$ at room temperature, the membranes were incubated overnight at $4^{\circ} \mathrm{C}$ with anti-TrkB $(1: 3000$, Cell Signaling). The membranes were probed with a mouse antiactin antibody as an internal control. The blots were then incubated for $1 \mathrm{~h}$ at room temperature with horseradish peroxidase-conjugated secondary antibody. Protein bands were detected using the ECL. Western Blotting Substrates (Pierce, Rockford, IL) were estimated using the Image Analysis Program Labwork 4.5. 


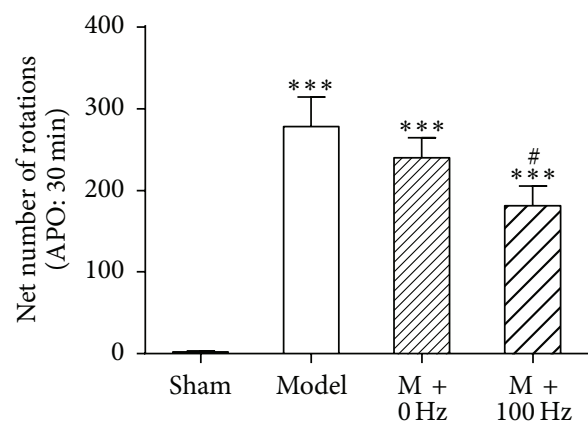

(a)

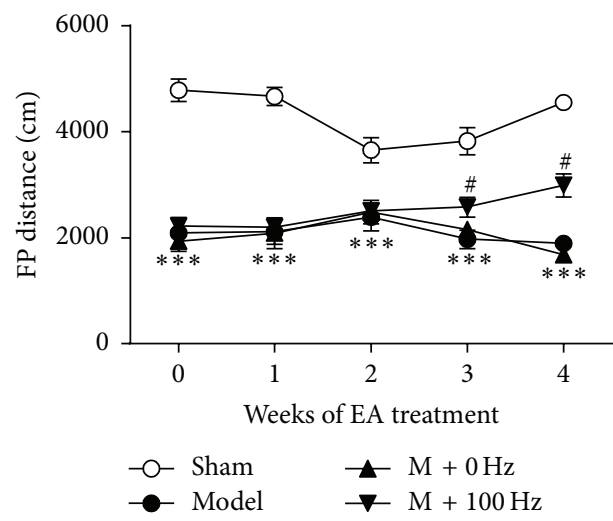

(c)

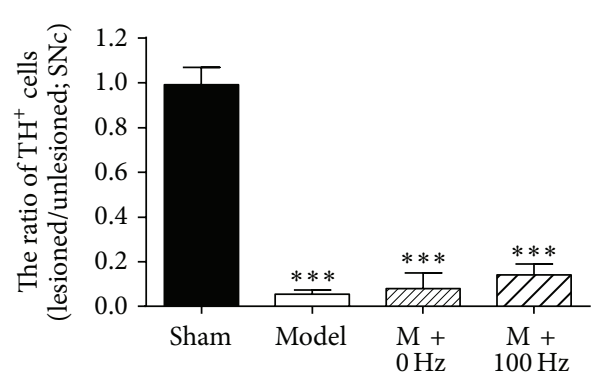

(e)

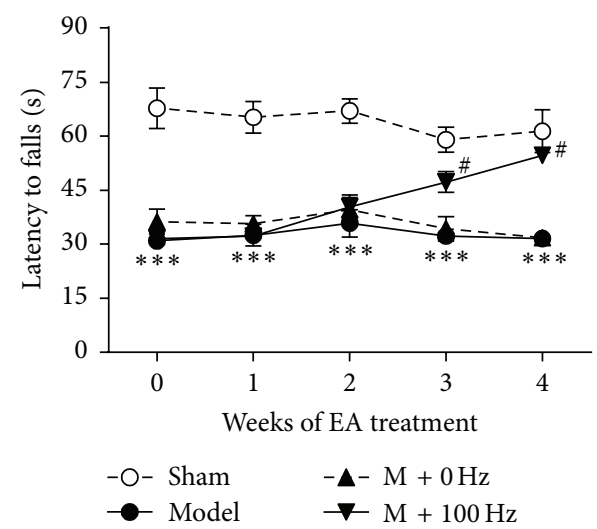

(b)

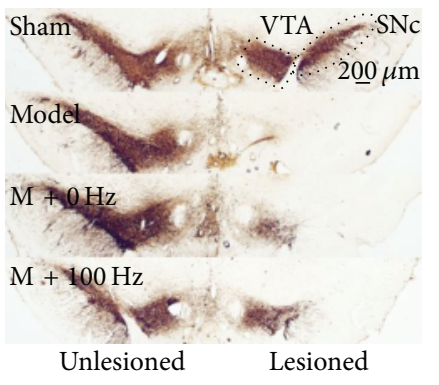

(d)

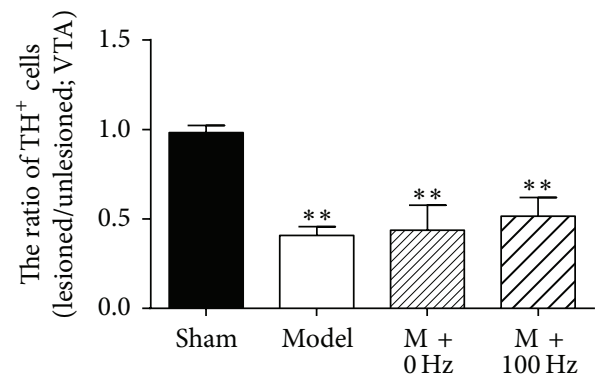

(f)

FIGURE 1: Effects of $100 \mathrm{~Hz}$ EA treatment on motor symptoms and dopaminergic neurodegeneration in the ventral midbrain of 6-OHDA lesioned rats. ((a)-(c)) Effects of EA on APO-induced rotational behavior (a), rotarod test (b), and horizontal movement distance in the open field test (c). Data represent mean \pm SEM ( $n=9-11$ per group). ${ }^{* *} P<0.01$ and ${ }^{* * *} P<0.001$ versus the sham group; ${ }^{*} P<0.05$ versus the model group. (d) Effects of EA on TH immunohistochemical staining. SNc is implied by the elliptical dotted line and VTA is implied by rectangle dotted line in (d). ((e) and (f)) Quantification of the damaged extent of TH-positive profiles on lesioned compared to unlesioned side in the SNc (e) and in the VTA (f). Data represent mean \pm SEM, ${ }^{* *} P<0.01$, and ${ }^{* * *} P<0.001$ versus the sham group.

2.8. Statistical Analysis. Data were reported as means \pm SEM. One- or two-way analysis of variance (ANOVA) was used, as appropriate, followed by the Newman-Keuls post hoc test for comparison of groups. $P<0.05$ was the criterion for statistical significance.

\section{Results}

3.1. Effects of EA on Motor Function and Dopaminergic Neurodegeneration in 6-OHDA Lesioned Rats. The apomorphineinduced turning test was performed 6 weeks after 6-OHDA lesions were induced in the rats. As shown in Figure 1(a), unilateral injection of 6-OHDA into the MFB produced intense contralateral turning behavior compared to the sham group $(P<0.05)$, while $100-\mathrm{Hz}$ EA treatment, but not 0 $\mathrm{Hz}$ EA treatment, reduced the intensity of apomorphineinduced turning. Sham animals $(n=19)$ showed no turning behavior. Motor coordination was assessed using the rotarod test (Figure 1(b)). A repeated two-way ANOVA showed an effect of group $\left(F_{3,34}=71.55 ; P<0.001\right)$ and weeks of treatment $\left(F_{4,34}=1.39 ; P=0.25\right)$ and an interaction of group $\times$ weeks $\left(F_{12,34}=4.53, P<0.001\right)$. Further post 
hoc analyses showed that 6-OHDA-lesioned rats developed significant coordination deficits $\left(F_{3,34}=24.35, P<0.001\right)$ before EA treatment. However, $100-\mathrm{Hz}$ EA did not reverse this latency after $1(P>0.05)$ or 2 weeks of treatment $(P>0.05)$ but showed some effect following $3(P<0.001)$ and 4 weeks $(P<0.001)$ of treatment compared with the 6-OHDA model group. Meanwhile, an obvious decrease in the horizontal movement distances was found in the 6OHDA group compared to the sham group $\left(F_{3,30}=34.86\right.$, $P<0.001)$ before EA treatment. However, $100-\mathrm{Hz}$ EA significantly increased horizontal movement distances after $3(P<0.001)$ and 4 weeks $(P<0.001)$ of treatment. Similar to previous reports, $0-\mathrm{Hz}$ EA showed no effect on these motor deficits [11]. These data suggest that long-term $100-\mathrm{Hz}$ EA stimulation might improve motor function in 6OHDA model rats.

Immunohistochemical staining for $\mathrm{TH}$ was performed 6 weeks after the MFB lesions were induced, and the numbers of TH-immunoreactive neurons in the SNc and VTA were quantified. The data show that 6-OHDA MFB lesions induced loss of TH-positive profiles by $94.5 \% \pm 1.8 \%$ in the $\mathrm{SNc}$ on the ipsilateral side compared with the unlesioned side $(P<0.001$, Figures $1(\mathrm{~d})$ and $1(\mathrm{e}))$. 100-Hz EA stimulation for 4 weeks showed no effects on the degeneration of TH-positive cells in the SNc compared with the 6-OHDA group $(P>0.05$, Figures $1(\mathrm{~d})$ and $1(\mathrm{e}))$. Noticeably, the TH-positive profiles decreased by $40.81 \% \pm 4.91 \%$ in the VTA of the lesioned side compared with the contralateral side (Figures $1(d)$ and $1(\mathrm{f}))$. However, an increase in TH-positive profiles in the ipsilateral VTA was observed after 4 weeks of $100-\mathrm{Hz}$ EA (Figure 1(f)), although this change was not significant $(P>$ $0.05)$. These results collectively support the contention that $100-\mathrm{Hz}$ EA can alleviate the abnormal movement symptoms triggered by 6-OHDA lesion and has a minimal impact on the mesocorticolimbic dopaminergic pathway but not on the mesostriatal pathway.

\subsection{Effects of EA on Depressive-Like Symptoms in 6-OHDA-} Lesioned Rats. Depressive-like behavioral phenotypes were assessed after 4 weeks of EA treatment. Anhedonic behavior was measured using the sucrose preference test. Two-way ANOVA showed significant effects of group $\left(F_{3,180}=14.62\right.$, $P<0.0001)$ and week $\left(F_{4,180}=2.62, P=0.0365\right)$ but no significant effect of the group $\times$ week interaction $\left(F_{12,180}=\right.$ $0.567, P=0.867)$. Bonferroni post hoc analysis shows that all 6-OHDA-lesioned rats displayed a significant decrease in sucrose solution intake 2 weeks after neurotoxin exposure compared to the sham rats $(P<0.05$; Figure $2(\mathrm{a}))$. The $100-$ $\mathrm{Hz}$ group showed a weekly increase in sucrose preference and a significant increase after $3(P<0.05)$ and 4 weeks $(P<0.05)$ of EA treatment compared with the 6-OHDA group.

FSTs were performed to evaluate behavioral despair in depressive rats. Six weeks after neurotoxin exposure, the 6OHDA group exhibited a significant increase in immobility time compared with the sham group $(P<0.05)$. However, 4 weeks of EA treatment effectively reduced immobility time compared to 6-OHDA group $(P<0.05)$. Analysis of swimming and climbing behavior in the FST demonstrated that
6-OHDA MFB lesions significantly affected these parameters (all $P<0.05$ ). Similarly, EA treatment effectively increased climbing time compared to 6-OHDA group $(P<0.05)$.

Moreover, the open field test was used to assess anxietyrelated behavior. Two-way ANOVA showed significant effects of group $\left(F_{3,124}=24.4, P<0.001\right)$ but no significant effects of week $\left(F_{3,124}=1.49, P=0.221\right)$ and interaction between group and week $\left(F_{9,124}=0.988, P=0.453\right)$ (Figure $2(\mathrm{c})$ ). In the open field test, 6-OHDA-lesioned rats showed fewer central square entries $(P<0.001$, Figure $2(\mathrm{c}))$ than sham rats. This result suggests that 6-OHDA induces an increase in anxiety-related behavior. However, the $100-\mathrm{Hz}$ EA group displayed an increase in the number of entries after $3(P<$ $0.05)$ and 4 weeks $(P<0.05)$ of treatment. Thus, continuous EA stimulation has a strong positive effect on reversing the anhedonic-like and despair-like behavioral phenotypes in 6OHDA-lesioned rats.

3.3. Effects of EA on the DA, 5-HT, and NE Levels in the Striatum and Hippocampus. To determine whether EA exerts its therapeutic effects by regulating neurotransmitters, the levels of DA, 5-HT, and NE in the striatum and hippocampus were measured after 4 weeks of $100-\mathrm{Hz}$ EA treatment. As shown in Figure 3(a), the level of DA was almost completely depleted in the 6-OHDA-lesioned ipsilateral striatum compared with that in the sham group $(P<0.001)$. Similarly, the DA has a tendencious but not significant decrease in the lesioned ipsilateral hippocampus compared with the level in the sham group $(P=0.095$, Figure $3(d))$. Neither level $100-\mathrm{Hz}$ nor level $0-\mathrm{Hz}$ EA produced any effect on the DA concentration either in the striatum (Figure 3(a)) or in the hippocampus (Figure 3(d)). The 5-HT and NE levels were also remarkably reduced in the lesioned hemisphere compared to the unlesioned hemisphere, both in the striatum $(P<0.001)$ and in the hippocampus $(P<0.001)$. Additionally, the 5-HT and NE levels in the striatum were unaffected by 4 weeks of treatment with 100-Hz EA (Figures 3(a), 3(b), and 3(c)), and similar results were observed in the hippocampus (Figures 3(d), 3(e), and 3(f)). These findings suggest that $100-\mathrm{Hz}$ EA has no influence on the levels of DA, 5-HT, and NE in the striatum and hippocampus.

3.4. Effects of EA Treatment on the Expression of BDNF and TrkB in the Hippocampus and Ventral Midbrain. To explore the possible mechanisms involved in the antidepressive effects of $100-\mathrm{Hz} \mathrm{EA}$, the rats were sacrificed after 4 weeks of EA treatment. As shown in Figures 4(a) and 4(c), 6-OHDA lesions induced an increased BDNF level $(P<0.05)$ on the lesioned side of ventral midbrain and a decreased BDNF level $(P<0.05)$ on the lesioned side of hippocampus compared with the sham group, respectively. However, these alterations in BDNF levels on the lesioned side induced by 6-OHDA were reversed by EA treatment both in the ventral midbrain and in the hippocampus. Although the changes in BDNF level on the unlesioned side were modulated by EA treatment in the ventral midbrain, it has no influence on the unlesioned side in the hippocampus (Figures 4(a) and $4(\mathrm{c})$ ). Meanwhile, there were significant decreases of 


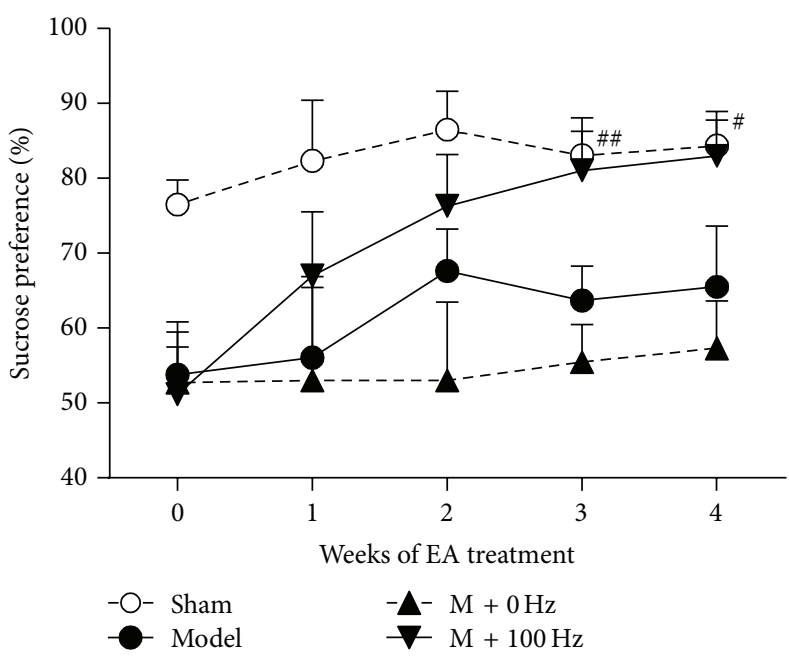

(a)

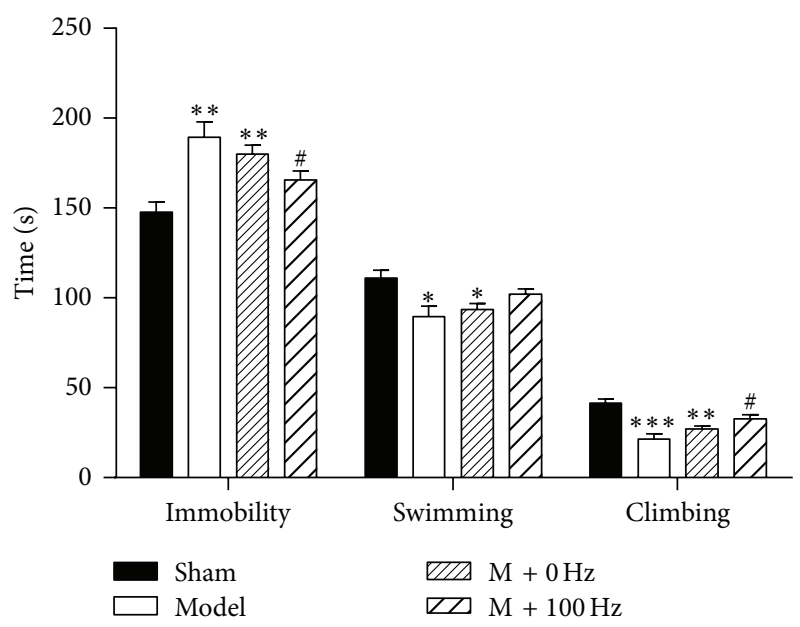

(b)

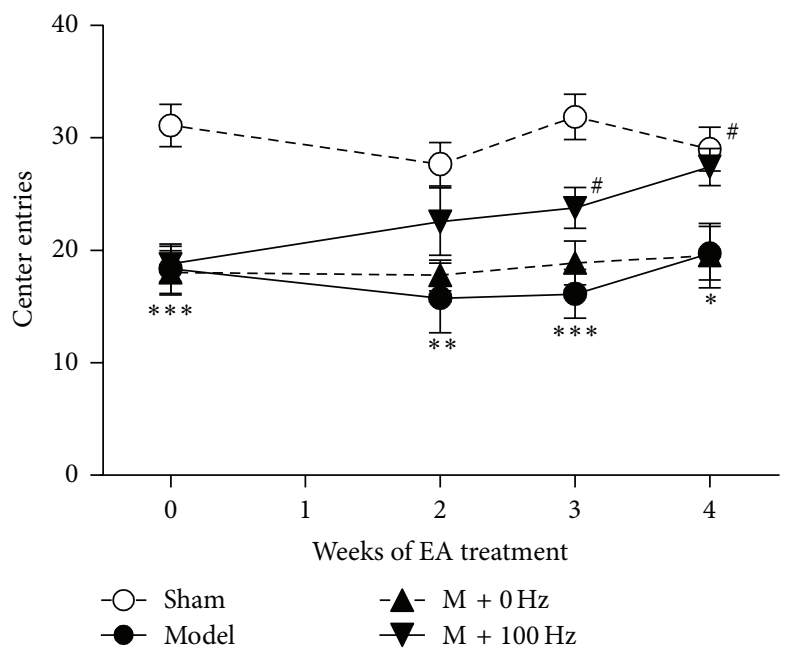

(c)

FIGURE 2: EA at $100 \mathrm{~Hz}$ improved depressive-like symptoms in 6-OHDA lesioned rats. (a) Changes of sucrose intake in sucrose preference test. (b) Changes of the immobility and swimming and climbing behaviors in the forced swim test. (c) Changes of the center entries in the open field test. Data represent mean $\pm \operatorname{SEM}\left(n=9-11\right.$ per group). ${ }^{*} P<0.05,{ }^{* *} P<0.01$, and ${ }^{* * *} P<0.001$ versus the sham group; ${ }^{*} P<0.05$ and ${ }^{\# \#} P<0.01$ versus the model group.

TrkB expression on the lesioned side both in the ventral midbrain and in the hippocampus compared with the sham group $(P<0.01) .100 \mathrm{~Hz}$ EA treatment could increase TrkB expression on the lesioned side both in the ventral midbrain and in the hippocampus (Figures 4(b) and 4(d)). Similarly, EA treatments have no obvious effects on the TrkB expression on the unlesioned side in the ventral midbrain or the hippocampus. These data suggest that $100-\mathrm{Hz}$ EA treatment could modulate 6-OHDA-induced abnormal expression of $\mathrm{BDNF}$ and TrkB on the lesioned side in the ventral midbrain and hippocampus.

\section{Discussion}

4.1. EA Improves Depressive Symptoms and Partly Affects VTA Dopaminergic Pathways. The core biochemical dysfunction that primarily, but not exclusively, contributes to the motor features in PD is the profound deficit in the DA level. When classic motor deficits (including resting tremor, rigidity, and bradykinesia) occur, there are more than $50 \%$ of dopaminergic neurons that are lost in the SNc, exceeding 60-80\% DA depletion in the striatum [34-37]. In the unilateral PD model used in the present study, almost all the dopaminergic neurons were lost in the SNc and the DA level was depleted 7-fold in the ipsilateral striatum after 6-OHDA lesions were induced in the MFB. The results of the apomorphine-induced rotation, open field, and rotarod tests strongly suggest that the motor hallmarks of PD are closely associated with mesostriatal dopaminergic degeneration and basal ganglia circuit dysfunction $[25,29]$.

Previous studies indicated that the 6-OHDA MFB lesion model is a relevant tool to study the pathophysiology of both the motor and nonmotor symptoms of PD, including severe motor dysfunction and depressive-like symptoms [29]. These 


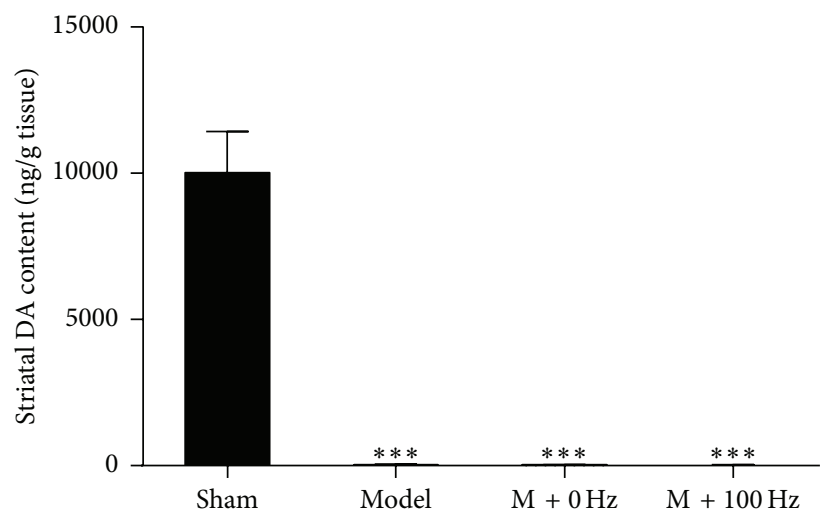

(a)

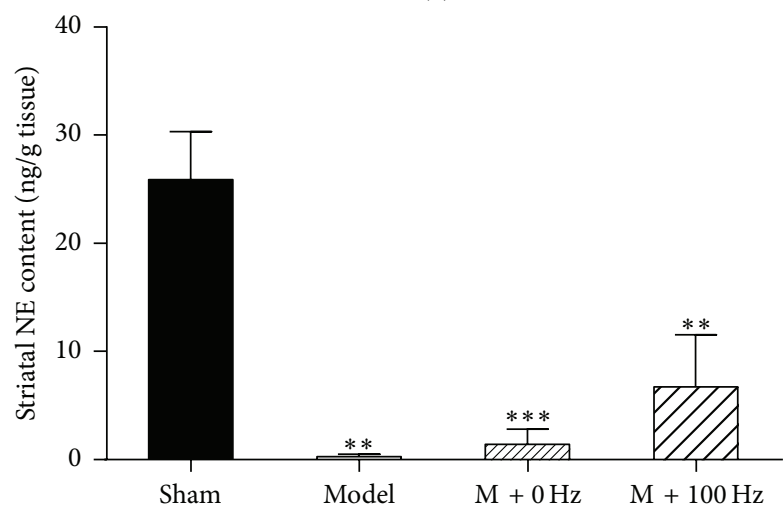

(b)

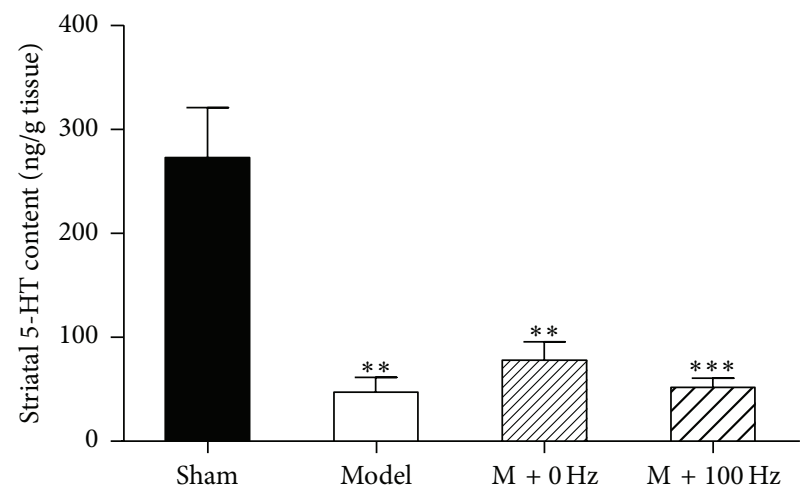

(c)

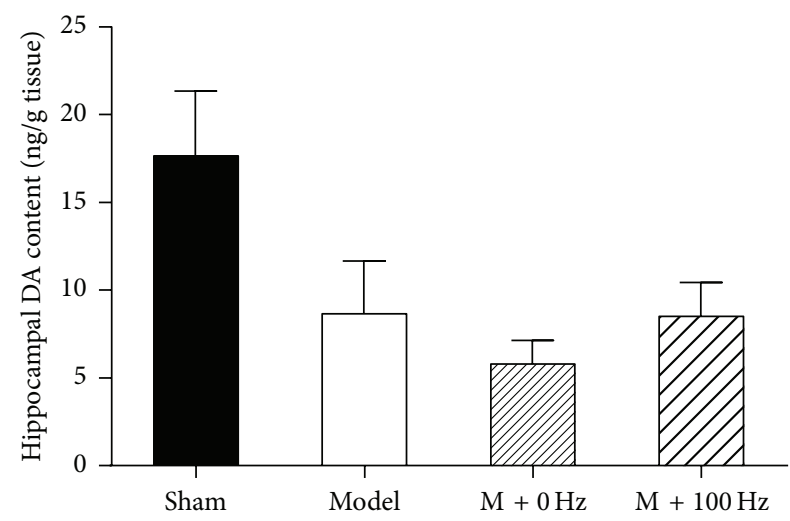

(d)

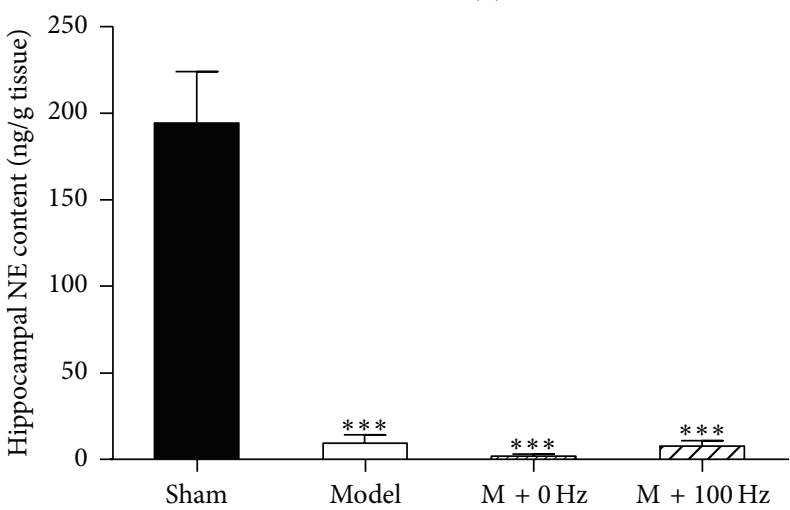

(e)

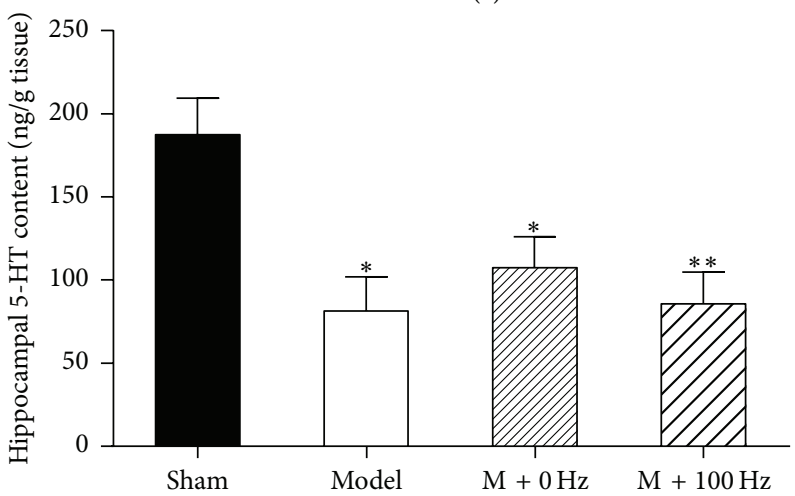

(f)

Figure 3: EA at $100 \mathrm{~Hz}$ had no significant effects on the contents of DA, NE, and 5-HT in the striatum ((a)-(c)) and that in the hippocampus $((\mathrm{d})-(\mathrm{f}))$. The content of transmitters was measured by HPLC. Data represent mean \pm SEM $\left(n=5-6\right.$ per group). ${ }^{*} P<0.05,{ }^{* *} P<0.01$, and ${ }^{* * *} P<0.001$ versus the sham group.

behavioral alterations can be attributed to the significant disruption in both the mesostriatal and mesocorticolimbic dopaminergic pathways. In the present experiment, the depressive symptoms of PD were measured using the sucrose preference test, which has been widely used to assess the anhedonic response in animal models of depression [38]. Two weeks after 6-OHDA administration, a significant decrease in sucrose consumption was found in the 6-OHDA-lesioned animals. The FST is a well-established behavioral assessment tool used to evaluate depressive-like features; particularly the prolonged immobility can be regarded as "behavioral despair." Our data showed that the 6-OHDA-lesioned model showed a high immobility time. Additionally, a significant reduction in dopaminergic neurons in the VTA and a tendencious but not significant decrease of the DA level in the hippocampus were found in the 6-OHDA-lesioned rats. Recent data suggest that damage to the dopaminergic neurons in the VTA affecting the ascending mesolimbic and mesocortical pathways leads to a depressive phenotype in rats and remarkable low swimming and climbing times. Meanwhile, the number of central square entries in the open field test was markedly reduced in the lesioned rats. 


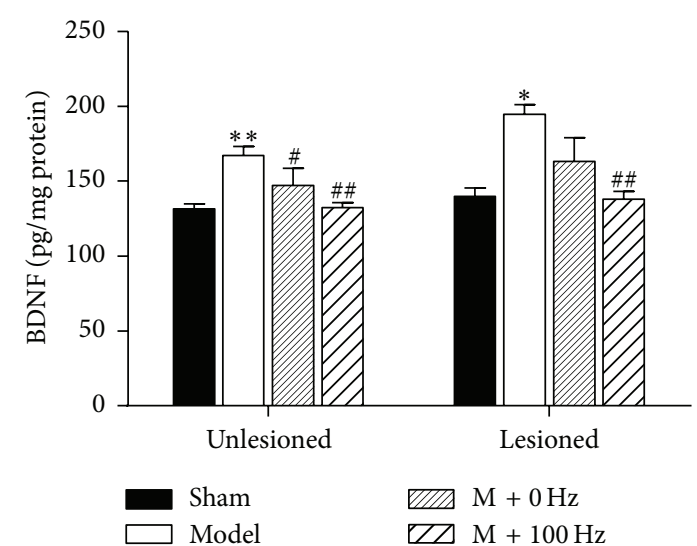

(a)
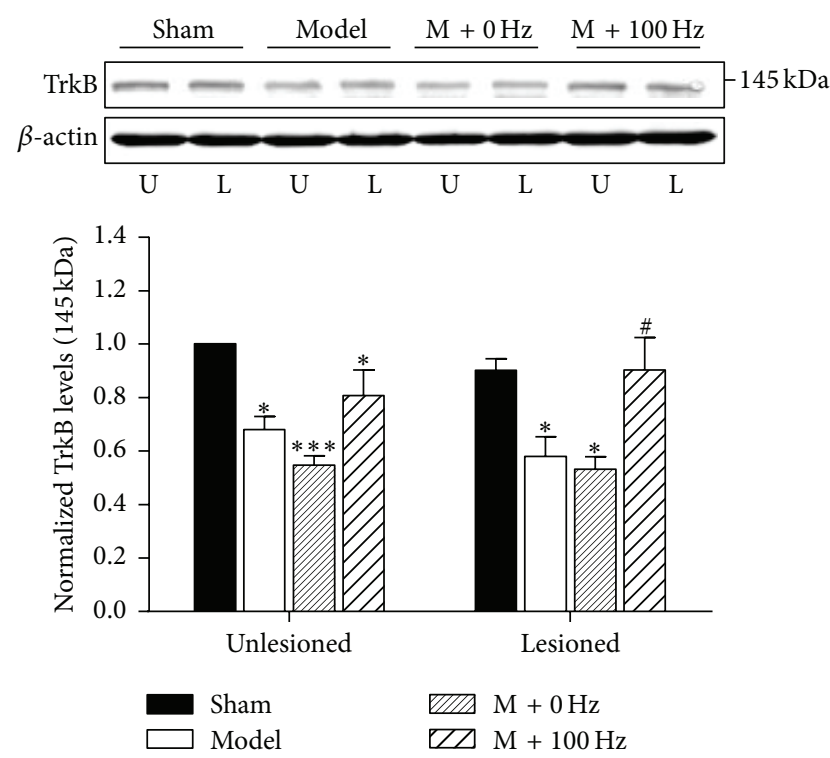

(b)

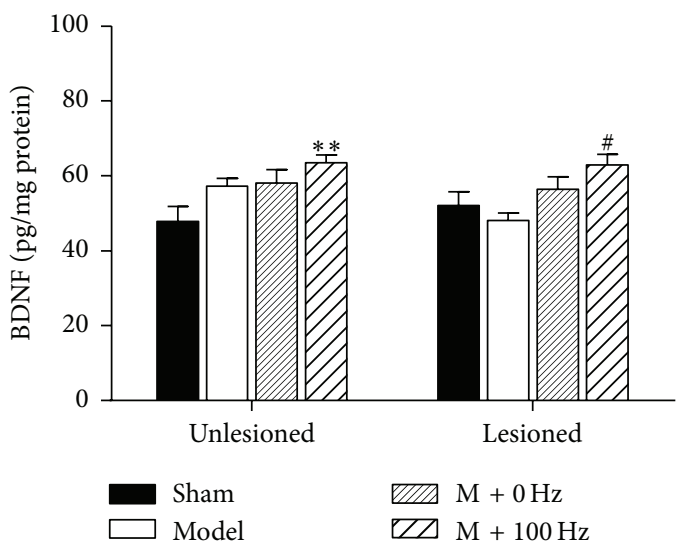

(c)
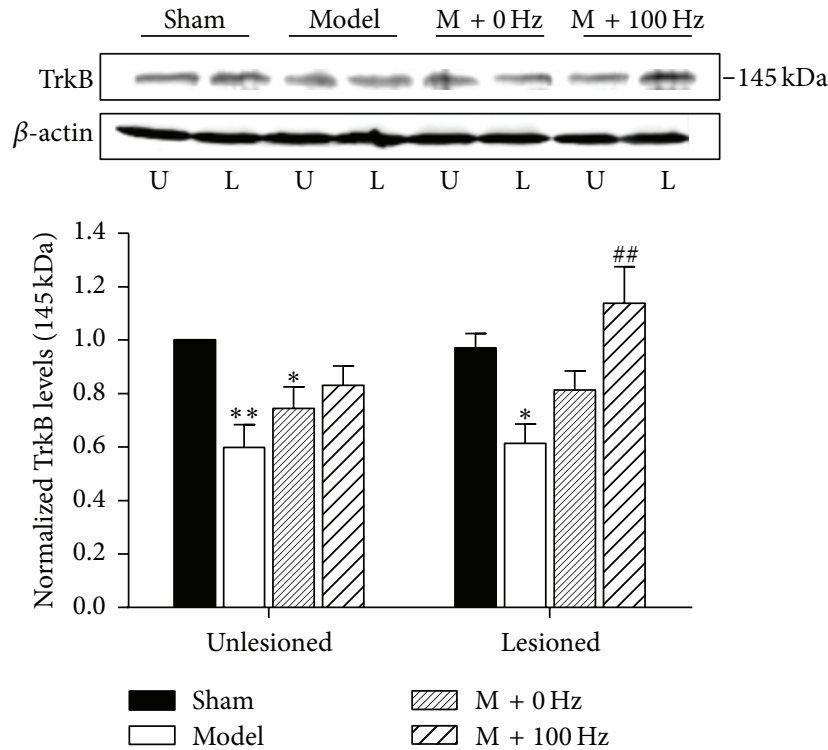

(d)

FIGURE 4: EA at $100 \mathrm{~Hz}$ modulated the abnormal expression of BDNF and TrkB in the midbrain and in the hippocampus. BDNF levels were measured by ELISA on the lesioned side and unlesioned side in the ventral midbrain (a) and the hippocampus (c), respectively. TrkB expressions were measured by western blotting on the unlesioned side and lesioned side in the ventral midbrain (b) and the hippocampus (d), respectively. $\beta$-actin was used as an internal control. Data represent mean \pm SEM $\left(n=5-6\right.$ per group). ${ }^{*} P<0.05$, ${ }^{* *} P<0.01$, and ${ }^{* * *} P<0.001$ versus the sham group; ${ }^{\#} P<0.05$ and ${ }^{\# \#} P<0.01$ versus the model.

Consistent with prior data [29], our results supported the contention that rats with complete unilateral dopaminergic neuron damage, that is, depletion of both mesocorticolimbic and nigrostriatal DA, develop severe motor impairments and depressive-like symptoms [28]. Clinical evidence has also shown that dopamine dysfunction in $\mathrm{PD}$-associated depression is mainly related to DA projections arising from neurons in the VTA [39]. In support of these data, previous neurochemical evidence has shown that mesencephalic dopaminergic projection to the hippocampus declined after 6-OHDA lesions in the SNc [40]. This situation mimics a mild damage of $\mathrm{PD}$ where mesencephalon-limbic dopaminergic pathways are affected [41]. Accordingly, HPLC analysis in the present study showed that the hippocampal content of DA in the 6-OHDA-lesioned animals was significantly reduced.
In the present study, we demonstrated that highfrequency EA improves the motor symptoms as well as the nonmotor symptoms in PD rats. Similar to our previous studies [11, 15], this study also showed that $100-\mathrm{Hz}$ EA alleviated abnormal movement symptoms but had no effect on nigrostriatal dopaminergic transmission. Interestingly, we found that EA produced a minimal neuroprotective impact on VTA dopaminergic neurons, although this result was not statistically significant. Thus, EA seems to affect mesencephalolimbic dopaminergic pathways and improve depressive symptoms.

4.2. EA Modulates BDNF-TrkB Signals but Has No Effect on the Noradrenergic and Serotonergic Systems. Although PD is widely known to be a disorder associated with the depletion 
of striatal DA, prior evidence has indicated that dysfunctions of other neurotransmitters, such as NE and 5-HT, also participate in the development of depression in PD [42]. According to the Braak staging of PD pathology, NA and 5HT dysfunction occur prior to the significant degradation of dopaminergic neurons [4].

Clinical evidence has also shown that 5-HT alterations occur in patients with PD-associated depression [36]. The administration of 6-OHDA in the MFB resulted in noradrenergic and serotonergic reduction in the striatum and hippocampus. On the basis of the known role of the serotonergic system in emotional processes, it is conceivable that reduced 5-HT levels are closely related to mood-related phenotypes [24]. The 6-OHDA-induced PD model produced sustained depressive-like behaviors, including anhedonia and behavioral despair, which are directly associated with the reduction in striatal DA and hippocampal 5-HT content [26]. In addition, decreased NE levels in the hippocampus and significant losses in limbic noradrenergic structures have also been found in PD patients [37]. Neuroimaging studies have shown that significant losses in limbic noradrenergic structures might be associated with depression in PD [31].

In the current study, we found that 6-OHDA induced a marked reduction in the levels of DA, 5-HT, and NE in both the striatum and hippocampus, which suggests that dysfunctions in these neurotransmitters may contribute to many nonmotor symptoms of PD. Thus, correlations may exist between swimming in the FST and the serotonergic system or between immobility in the FST and the dopaminergic system. Therefore, one or more of these neurotransmitter systems may play an important role in depressive-like behavior in this PD model, and interventions aimed at restoring monoamine function may be beneficial in treating the disease [24, 36]. However, neither $100-\mathrm{Hz}$ nor $0-\mathrm{Hz}$ EA elicited any significant changes in the content of DA, 5-HT, or NE either in the striatum or in the hippocampus in the 6-OHDA-lesioned rats. Therefore, the mechanism of action of EA treatment does not seem to be related to these neurotransmitters.

As a key regulator of synaptic development and plasticity, BDNF has been recognized to play a pivotal role in several neurodegenerative and psychiatric disorders [24]. Our previous experiment suggested that high-frequency EA plays a neurotrophic role in PD rats by increasing BDNF mRNA levels in the SNc and VTA [15]. The protective properties of acupuncture might be mediated by TrkB receptors in the 6OHDA model [43]. Thus, our study revealed the regulatory effect of EA on BDNF-TrkB signaling.

Correlations between depressive-like symptoms and the reduction in BDNF $\mathrm{mRNA}$ and protein levels in the hippocampus have been identified in postmortem evidence [16, 17]. Further, enhanced BDNF expression has been found in the nucleus accumbens of individuals with depression [44]. These alterations were reversed following antidepressant treatment $[45,46]$. In experimental models, BDNF levels decreased in the hippocampus and frontal cortex $[20,21]$, whereas they increased in the nucleus accumbens and VTA [22, 47], which might suggest different roles of BDNF in different brain structures [27]. Similarly, we also observed a decrease in BDNF levels and TrkB expression in the hippocampus after 6-OHDA injection. Notably, 4 weeks of EA treatment remarkably increased the BDNF levels and TrkB expression in the hippocampus. Thus, the antidepressant role of EA may activate a cascade of neurotrophic activity including BDNF and $\operatorname{TrkB}$ within the mesocorticolimbic dopaminergic system, which originates from the ventral tegmental area and innervates the thalamus and hippocampus.

In contrast, 6-OHDA lesions induced an increase in BDNF expression in the midbrain. There are some complex reasons as to why this may be. Because BDNF is highly expressed by dopaminergic neurons, the degeneration of dopaminergic neurons should induce decreased BDNF expression. However, it has been previously shown that 6-OHDA injection triggers microglia and astrocyte proliferation and increases BDNF-immunoreactivity in the lesioned midbrain $[48,49]$. In addition, the SNc and VTA can project to the hippocampus through the activation of $5-\mathrm{HT}_{2 \mathrm{~A}}$ receptors to alter serotonin transmission [50]. Therefore, there should be a correlation between the reduced BDNF expression in the hippocampus and increased BDNF expression in the midbrain. Moreover, decreased expression of TrkB in the midbrain was also observed in the 6-OHDA model [43]. This discrepancy between the changes in BDNF and TrkB levels has not been clarified thus far and requires further investigation.

In the present study, alterations in the BDNF levels and $\operatorname{TrkB}$ expression induced by 6-OHDA lesions were reversed by EA treatment in the hippocampus and midbrain. Therefore, we concluded that EA might modulate BDNFTrkB interaction in the mesolimbic dopaminergic pathway. Moreover, BDNF seems to participate in the effects of EA and may serve as a potential therapeutic target in PD.

\section{Conclusion}

In summary, we demonstrated that high-frequency EA improves the motor symptoms as well as the nonmotor symptoms in PD rats. The effects of EA in improving depressivelike symptoms might be partially attributed to the neuroprotective role of EA in the mesocorticolimbic dopaminergic pathways. $100-\mathrm{Hz}$ EA did not elicit any significant changes in the level of 5-HT and NE either in the striatum or in the hippocampus of the 6-OHDA-lesioned rats. Interestingly, BDNF-TrkB pathway seems to participate in the effect of EA and may serve as potential therapeutic targets in PD.

\section{Competing Interests}

The authors declare that there is no conflict of interests regarding the publication of this paper.

\section{Acknowledgments}

This study was supported by the National Natural Science Foundation of China (81072858, 81473770, and 81030062), the Importation and Development of High-Caliber Talents 
Project of Beijing Municipal Institutions (CIT\&TCD201304185) and the Project of Construction of Innovative Teams and Teacher Career Development for Universities and Colleges under Beijing Municipality (IDHT20140514), and the Seed Grant of International Alliance of Translational Neuroscience (PXM2014_014226_000015). The authors express their sincere thanks to Dr. Wang Qiang (University of MissouriKansas City) for his critical readings of the paper.

\section{References}

[1] M. M. S. Lima, E. F. Martins, A. M. Delattre et al., "Motor and non-motor features of Parkinson's disease-a review of clinical and experimental studies," CNS and Neurological DisordersDrug Targets, vol. 11, no. 4, pp. 439-449, 2012.

[2] K. Seppi, D. Weintraub, M. Coelho et al., "The movement disorder society evidence-based medicine review update: treatments for the non-motor symptoms of Parkinson's disease," Movement Disorders, vol. 26, no. 3, pp. S42-S80, 2011.

[3] S. Papapetropoulos and D. C. Mash, "Psychotic symptoms in Parkinson's disease. From description to etiology," Journal of Neurology, vol. 252, no. 7, pp. 753-764, 2005.

[4] J. S. A. M. Reijnders, U. Ehrt, W. E. J. Weber, D. Aarsland, and A. F. G. Leentjens, "A systematic review of prevalence studies of depression in Parkinson's disease," Movement Disorders, vol. 23, no. 2, pp. 183-189, 2008.

[5] F. L. Rocha, M. G. R. Murad, B. P. Stumpf, C. Hara, and C. Fuzikawa, "Antidepressants for depression in Parkinson's disease: systematic review and meta-analysis," Journal of Psychopharmacology, vol. 27, no. 5, pp. 417-423, 2013.

[6] F. J. E. Vajda and C. Solinas, "Current approaches to management of depression in Parkinson's disease," Journal of Clinical Neuroscience, vol. 12, no. 7, pp. 739-743, 2005.

[7] L. M. Shulman, X. Wen, W. J. Weiner et al., "Acupuncture therapy for the symptoms of Parkinson's disease," Movement Disorders, vol. 17, no. 4, pp. 799-802, 2002.

[8] D. V. Arankalle and P. M. K. Nair, "Effect of electroacupuncture on function and quality of life in Parkinson's disease: a case report," Acupuncture in Medicine, vol. 31, no. 2, pp. 235-238, 2013.

[9] Y. Xia, H.-D. Wang, Y. Ding, B. Kang, and W.-G. Liu, "Parkinson's disease combined with depression treated with electroacupuncture and medication and its effect on serum BDNF," Zhongguo Zhen Jiu, vol. 32, no. 12, pp. 1071-1074, 2012.

[10] F. Wang, L. Sun, X.-Z. Zhang et al., "Effect and potential mechanism of electroacupuncture add-on treatment in patients with Parkinson's disease," Evidence-Based Complementary and Alternative Medicine, vol. 2015, Article ID 692795, 11 pages, 2015.

[11] J. Jia, Z. Sun, B. Li et al., "Electro-acupuncture stimulation improves motor disorders in Parkinsonian rats," Behavioural Brain Research, vol. 205, no. 1, pp. 214-218, 2009.

[12] H. Wang, Y. Pan, B. Xue et al., "The antioxidative effect of electro-acupuncture in a mouse model of Parkinson's disease," PLoS ONE, vol. 6, no. 5, Article ID e19790, 2011.

[13] J. Deng, E. Lv, J. Yang et al., "Electroacupuncture remediates glial dysfunction and ameliorates neurodegeneration in the astrocytic $\alpha$-synuclein mutant mouse model," Journal of Neuroinflammation, vol. 12, no. 1, article 103, 2015.
[14] X.-Y. Liu, H.-F. Zhou, Y.-L. Pan et al., "Electro-acupuncture stimulation protects dopaminergic neurons from inflammation-mediated damage in medial forebrain bundle-transected rats," Experimental Neurology, vol. 189, no. 1, pp. 189-196, 2004.

[15] X.-B. Liang, X.-Y. Liu, F.-Q. Li et al., "Long-term high-frequency electro-acupuncture stimulation prevents neuronal degeneration and up-regulates BDNF mRNA in the substantia nigra and ventral tegmental area following medial forebrain bundle axotomy," Molecular Brain Research, vol. 108, no. 1-2, pp. 51-59, 2002.

[16] D. W. Howells, M. J. Porritt, J. Y. F. Wong et al., "Reduced BDNF mRNA expression in the Parkinson's disease substantia nigra," Experimental Neurology, vol. 166, no. 1, pp. 127-135, 2000.

[17] E. Shimizu, K. Hashimoto, N. Okamura et al., "Alterations of serum levels of brain-derived neurotrophic factor (BDNF) in depressed patients with or without antidepressants," Biological Psychiatry, vol. 54, no. 1, pp. 70-75, 2003.

[18] F. Karege, G. Vaudan, M. Schwald, N. Perroud, and R. La Harpe, "Neurotrophin levels in postmortem brains of suicide victims and the effects of antemortem diagnosis and psychotropic drugs," Molecular Brain Research, vol. 136, no. 1-2, pp. 29-37, 2005.

[19] L. Song, W. Che, W. Min-Wei, Y. Murakami, and K. Matsumoto, "Impairment of the spatial learning and memory induced by learned helplessness and chronic mild stress," Pharmacology Biochemistry and Behavior, vol. 83, no. 2, pp. 186-193, 2006.

[20] Q.-Q. Mao, Z. Huang, X.-M. Zhong, Y.-F. Xian, and S.-P. Ip, "Brain-derived neurotrophic factor signalling mediates the antidepressant-like effect of piperine in chronically stressed mice," Behavioural Brain Research, vol. 261, pp. 140-145, 2014.

[21] M. A. Smith, S. Makino, R. Kvetnansky, and R. M. Post, "Stress and glucocorticoids affect the expression of brain-derived neurotrophic factor and neurotrophin-3 mRNAs in the hippocampus," The Journal of Neuroscience, vol. 15, no. 3, pp. 17681777, 1995.

[22] J. M. Bessa, M. Morais, F. Marques et al., "Stress-induced anhedonia is associated with hypertrophy of medium spiny neurons of the nucleus accumbens," Translational Psychiatry, vol. 3, article e266, 2013.

[23] E. Castrén and T. Rantamäki, "The role of BDNF and its receptors in depression and antidepressant drug action: reactivation of developmental plasticity," Developmental Neurobiology, vol. 70, no. 5, pp. 289-297, 2010.

[24] M. T. Tadaiesky, P. A. Dombrowski, C. P. Figueiredo, E. CargninFerreira, C. Da Cunha, and R. N. Takahashi, "Emotional, cognitive and neurochemical alterations in a premotor stage model of Parkinson's disease," Neuroscience, vol. 156, no. 4, pp. 830-840, 2008.

[25] C. Winter, A. Von Rumohr, A. Mundt et al., "Lesions of dopaminergic neurons in the substantia nigra pars compacta and in the ventral tegmental area enhance depressive-like behavior in rats," Behavioural Brain Research, vol. 184, no. 2, pp. 133-141, 2007.

[26] R. M. Santiago, J. Barbiero, R. W. Gradowski et al., "Induction of depressive-like behavior by intranigral 6-OHDA is directly correlated with deficits in striatal dopamine and hippocampal serotonin," Behavioural Brain Research, vol. 259, pp. 70-77, 2014.

[27] K. Berghauzen-Maciejewska, J. Wardas, B. Kosmowska, U. Głowacka, K. Kuter, and K. Ossowska, "Alterations of BDNF and trkB mRNA expression in the 6-hydroxydopamine-induced model of preclinical stages of Parkinson's disease: an influence 
of chronic pramipexole in rats," PLoS ONE, vol. 10, no. 3, Article ID e0117698, 2015.

[28] L. L. Furlanetti, V. A. Coenen, and M. D. Döbrössy, "Ventral tegmental area dopaminergic lesion-induced depressive phenotype in the rat is reversed by deep brain stimulation of the medial forebrain bundle," Behavioural Brain Research, vol. 299, pp. 132-140, 2016.

[29] M. M. Carvalho, F. L. Campos, B. Coimbra et al., "Behavioral characterization of the 6-hydroxidopamine model of Parkinson's disease and pharmacological rescuing of non-motor deficits," Molecular Neurodegeneration, vol. 8, no. 1, article 14, pp. 1-11, 2013.

[30] L. L. Furlanetti, V. A. Coenen, I. A. Aranda, and M. D. Döbrössy, "Chronic deep brain stimulation of the medial forebrain bundle reverses depressive-like behavior in a hemiparkinsonian rodent model," Experimental Brain Research, vol. 233, no. 11, pp. 30733085, 2015.

[31] P. Remy, M. Doder, A. Lees, N. Turjanski, and D. Brooks, "Depression in Parkinson's disease: loss of dopamine and noradrenaline innervation in the limbic system," Brain, vol. 128, no. 6, pp. 1314-1322, 2005.

[32] J. Yang, Y. Pei, Y.-L. Pan et al., "Enhanced antidepressant-like effects of electroacupuncture combined with citalopram in a rat model of depression," Evidence-Based Complementary and Alternative Medicine, vol. 2013, Article ID 107380, 12 pages, 2013.

[33] Y. Yu, K. Wang, J. Deng et al., "Electroacupuncture produces the sustained motor improvement in 6-hydroxydopamine-lesioned mice," PLoS ONE, vol. 11, no. 2, Article ID e0149111, 2016.

[34] H. Bernheimer, W. Birkmayer, O. Hornykiewicz, K. Jellinger, and F. Seitelberger, "Brain dopamine and the syndromes of Parkinson and Huntington. Clinical, morphological and neurochemical correlations," Journal of the Neurological Sciences, vol. 20, no. 4, pp. 415-455, 1973.

[35] W. Dauer and S. Przedborski, "Parkinson's disease: mechanisms and models," Neuron, vol. 39, no. 6, pp. 889-909, 2003.

[36] A. Schrag, "Psychiatric aspects of Parkinson's disease: an update," Journal of Neurology, vol. 251, no. 7, pp. 795-804, 2004.

[37] B. Scatton, F. Javoy-Agid, L. Rouquier, B. Dubois, and Y. Agid, "Reduction of cortical dopamine, noradrenaline, serotonin and their metabolites in Parkinson's disease," Brain Research, vol. 275, no. 2, pp. 321-328, 1983.

[38] T. K. S. Craft and A. C. Devries, "Role of IL-1 in poststroke depressive-like behavior in mice," Biological Psychiatry, vol. 60, no. 8, pp. 812-818, 2006.

[39] A. Lieberman, "Depression in Parkinson's disease-a review," Acta Neurologica Scandinavica, vol. 113, no. 1, pp. 1-8, 2006.

[40] S. Maisonnette, J. P. Huston, M. Brandao, and R. K. W. Schwarting, "Behavioral asymmetries and neurochemical changes after unilateral lesions of tuberomammillary nucleus or substantia nigra," Experimental Brain Research, vol. 120, no. 3, pp. 273-282, 1998.

[41] E. Miyoshi, S. Wietzikoski, M. Camplessei, R. Silveira, R. N. Takahashi, and C. Da Cunha, "Impaired learning in a spatial working memory version and in a cued version of the water maze in rats with MPTP-induced mesencephalic dopaminergic lesions," Brain Research Bulletin, vol. 58, no. 1, pp. 41-47, 2002.

[42] A. L. Coppell, Q. Pei, and T. S. C. Zetterström, "Bi-phasic change in BDNF gene expression following antidepressant drug treatment," Neuropharmacology, vol. 44, no. 7, pp. 903-910, 2003.

[43] H.-J. Park, S. Lim, W.-S. Joo et al., "Acupuncture prevents 6hydroxydopamine-induced neuronal death in the nigrostriatal dopaminergic system in the rat Parkinson's disease model," Experimental Neurology, vol. 180, no. 1, pp. 93-98, 2003.

[44] V. Krishnan, M.-H. Han, D. L. Graham et al., "Molecular adaptations underlying susceptibility and resistance to social defeat in brain reward regions," Cell, vol. 131, no. 2, pp. 391-404, 2007.

[45] O. Aydemir, A. Deveci, and F. Taneli, "The effect of chronic antidepressant treatment on serum brain-derived neurotrophic factor levels in depressed patients: a preliminary study," Progress in Neuro-Psychopharmacology \& Biological Psychiatry, vol. 29, no. 2, pp. 261-265, 2005.

[46] N. Gervasoni, J.-M. Aubry, G. Bondolfi et al., "Partial normalization of serum brain-derived neurotrophic factor in remitted patients after a major depressive episode," Neuropsychobiology, vol. 51, no. 4, pp. 234-238, 2005.

[47] A. J. Eisch, C. A. Bolaños, J. De Wit et al., "Brain-derived neurotrophic factor in the ventral midbrain-nucleus accumbens pathway: a role in depression," Biological Psychiatry, vol. 54, no. 10, pp. 994-1005, 2003.

[48] C. Knott, G. Stern, A. Kingsbury, A. A. Welcher, and G. P. Wilkin, "Elevated glial brain-derived neurotrophic factor in Parkinson's diseased nigra," Parkinsonism and Related Disorders, vol. 8, no. 5, pp. 329-341, 2002.

[49] S. R. W. Stott and R. A. Barker, "Time course of dopamine neuron loss and glial response in the 6-OHDA striatal mouse model of Parkinson's disease," European Journal of Neuroscience, vol. 39, no. 6, pp. 1042-1056, 2014.

[50] M. Pierucci, V. Di Matteo, A. Benigno, G. Crescimanno, E. Esposito, and G. Di Giovanni, "The unilateral nigral lesion induces dramatic bilateral modification on rat brain monoamine neurochemistry," Annals of the New York Academy of Sciences, vol. 1155, no. 1, pp. 316-323, 2009. 


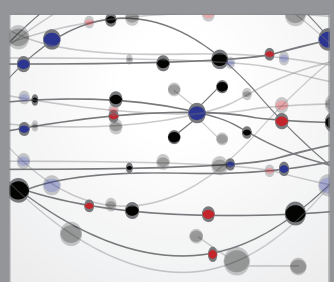

The Scientific World Journal
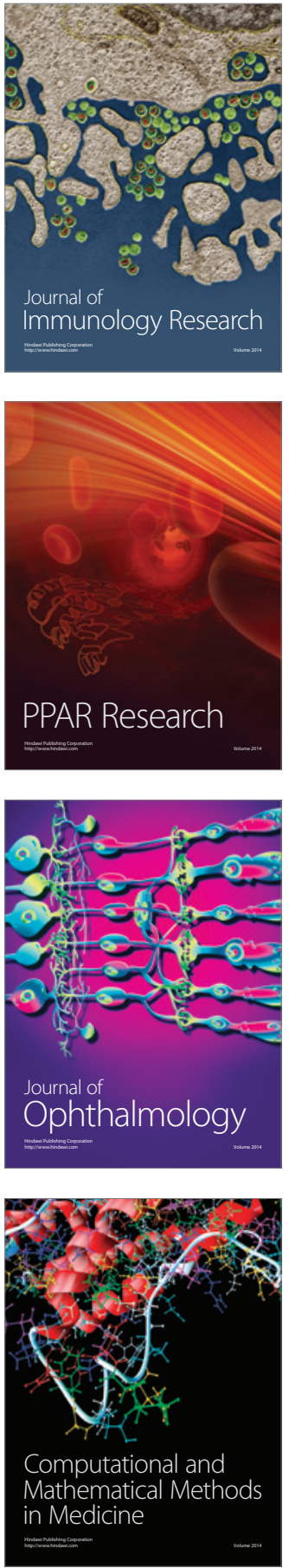

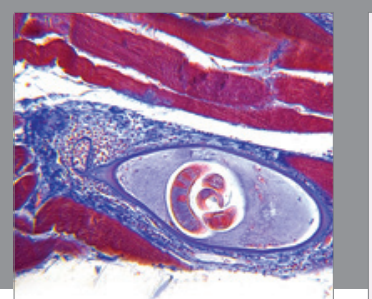

Gastroenterology Research and Practice

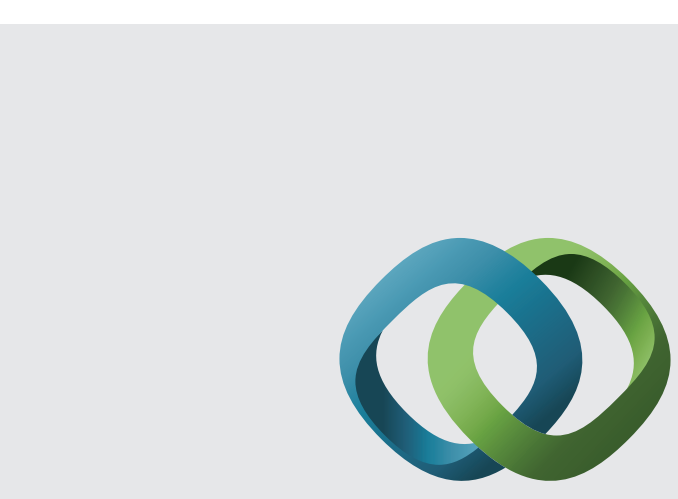

\section{Hindawi}

Submit your manuscripts at

http://www.hindawi.com
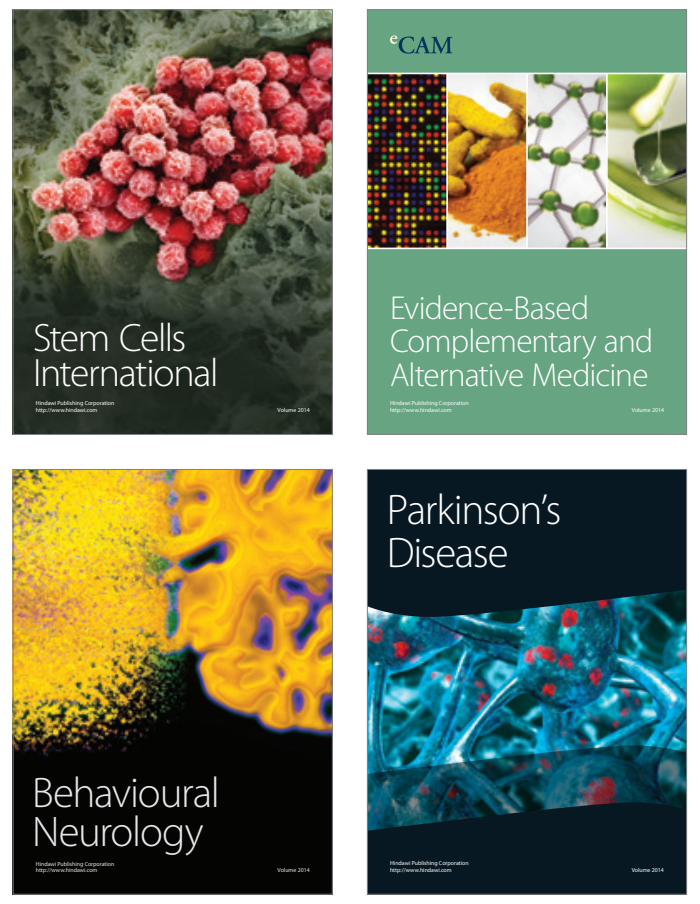
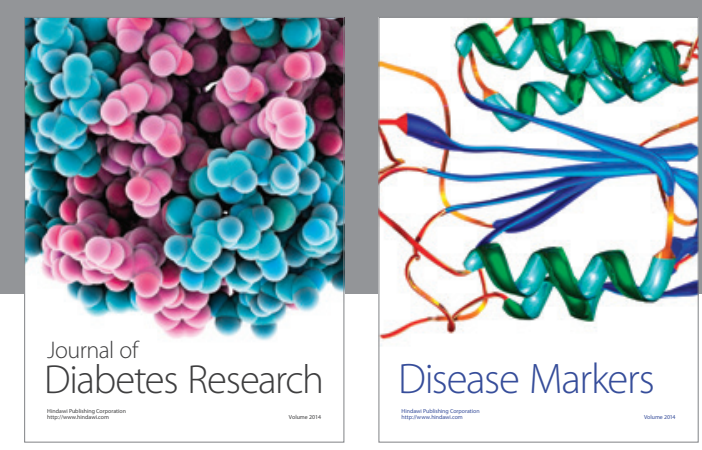

Disease Markers
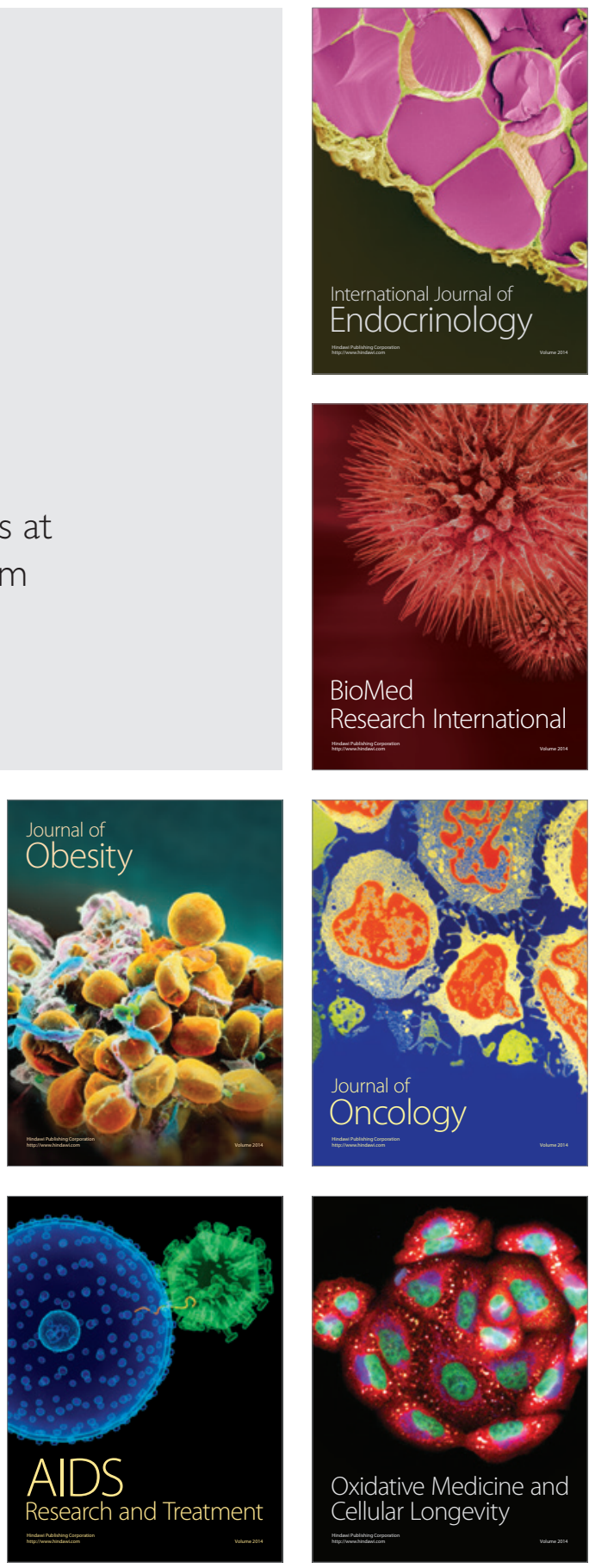